\title{
Changes in levels of selected incretins and appetite-controlling hormones following surgical treatment for morbid obesity
}

\author{
Piotr Major ${ }^{1}$, Maciej Matłok ${ }^{1}$, Michał Pędziwiatr ${ }^{1}$, Marcin Migaczewski ${ }^{1}$, Anna Zub-Pokrowiecka ${ }^{1}$, Dorota Radkowiak ${ }^{1}$, \\ Marek Winiarski ${ }^{1}$, Anna Zychowicz ${ }^{1}$, Danuta Fedak ${ }^{2}$, Andrzej Budzyński ${ }^{1}$ \\ ${ }^{1} 2^{\text {nd }}$ Department of Surgery, Jagiellonian University Medical College, Krakow, Poland \\ ${ }^{2}$ Department of Clinical Biochemistry, Jagiellonian University Medical College, Krakow, Poland
}

Videosurgery Miniinv 2015; 10 (3): 458-465

DOI: $10.5114 /$ wiitm.2015.54003

\begin{abstract}
Introduction: The hormonal brain-gut axis is a crucial element in appetite control and the response to surgical treatment for super obesity. However, mechanisms underlying the metabolic response to surgical treatment for morbid obesity are still not clearly specified.

Aim: To evaluate and compare the effects of surgical treatment for super obesity by laparoscopic sleeve gastrectomy (LSG) and by laparoscopic Roux-en-Y gastric bypass (LRYGB) on selected incretins and appetite-controlling hormones. Material and methods: Thirty-five patients were enrolled in a prospective study. Laparoscopic sleeve gastrectomy was performed in $45.8 \%$ of patients, and $L R Y G B$ in the remaining $54.2 \%$ of patients. Before the procedure fasting blood serum was collected from patients and preserved, to determine levels of selected incretins and brain-gut hormones: glucagon-like peptide 1 (GLP-1), peptide YY (PYY), leptin, and ghrelin.

Results: Twenty-eight patients came to a follow-up visit 12 months after the surgery. In these patients selected parameters were determined again. The percentage weight loss was $58.8 \%$. The ghrelin levels had decreased, and no statistically significant difference was observed between the two procedures. After both surgical procedures a statistically significant reduction in the leptin level was also observed. Peptide YY levels statistically significantly increased in the whole studied group. The GLP-1 level increased after the surgical procedure. However, the observed change was not statistically significant.

Conclusions: Both treatment methods result in modification of secretion patterns for selected gastrointestinal hormones, and this was considered to be a beneficial effect of bariatric treatment. The laparoscopic sleeve gastrectomy, being a procedure resulting in a metabolic response, seems to be an equally effective method for treatment of super obesity and comorbidities as the laparoscopic gastric bypass.
\end{abstract}

Key words: bariatric surgery, laparoscopic sleeve gastrectomy (LSG), Roux-en-Y gastric bypass (RYGB), ghrelin, leptin, glucagon-like peptide 1.

\section{Introduction}

Prevalence of obesity is increasing rapidly both in developed and developing countries, already reaching epidemic levels [1]. Currently, bariatric procedures are considered to be the sole proven method for permanent body weight reduction in the majority of treated patients, and achieve the highest remission rate for comorbidities [2].

Previously, the restriction effect resulting in reduction of possible food intake and limited food absorption were considered to be the main mechanisms underlying the decrease in body weight following bariatric procedures. So far, over twenty

\section{Address for correspondence}

Piotr Major MD, PhD, $2^{\text {nd }}$ Department of Surgery, Jagiellonian University Medical College, 21 Kopernika St, 31-501 Krakow, Poland,

phone: +48 693313 948, e-mail: majorpiotr@gmail.com 
hormonally active substances have been identified, secreted by gastrointestinal structures and participating in mechanisms controlling hunger and satiation. Changes in their secretion are considered to be responsible for metabolic effects of bariatric procedures [3]. Therefore, the hormonal brain-gut axis is a crucial component in appetite control and a response to surgical treatment for super obesity [4]. Adaptive changes in secretion of those crucial substances lead to body weight reduction and beneficial metabolic effects [5]. Although there are numerous publications available studying the effect of bariatric procedures on changes in hormonal axes controlling appetite, the mechanisms underlying a metabolic response to surgical treatment for super obesity are not yet fully known and clearly defined. Studies comparing the effect of individual surgical procedures on changes in selected hormones and incretins in the gastrointestinal tract are also lacking, and they would support better qualification of patients for an appropriate treatment method [6].

\section{Aim}

The aim of this study is to evaluate and compare the effects of surgical treatment for morbid obesity by laparoscopic sleeve gastrectomy (LSG) and by laparoscopic gastric bypass on selected incretins and appetite-controlling hormones.

\section{Material and methods}

A prospective study was conducted in a group of patients with super obesity undergoing operations at the $2^{\text {nd }}$ Chair of Surgery, Jagiellonian University Medical College (JUMC).

Criteria for enrolment in the study group:

- patients with super obesity meeting qualification criteria for bariatric surgery developed by the Metabolic and Bariatric Surgery Section of the Polish Surgical Society, i.e.:

- body mass index (BMI) exceeding $40 \mathrm{~kg} / \mathrm{m}^{2}$,

- BMI exceeding $35 \mathrm{~kg} / \mathrm{m}^{2}$ when obesity-related diseases are diagnosed, including type 2 diabetes, hypertension, lipid disorders, obstructive sleep apnoea,

- age 18-65 years;

- patients subjected to the LSG or the laparoscopic Roux-en-Y gastric bypass;

- postoperative period without complications;

- written consent to participate in the study.
Patients who: underwent revision surgery, suffered delayed surgical complications, did not report to a follow-up visit 12 months after the surgery, were diagnosed with mental illness, were diagnosed with alcohol or drug dependence were excluded from the study group.

Before the procedure, body weight and BMI were determined in all patients included in the study, using the TANITA BC-420S MA apparatus. Furthermore, information of super obesity complications (hypertension and type 2 diabetes) was collected. On the day of the procedure, in the morning, fasting blood serum was collected from patients and preserved, to determine levels of selected incretins and brain-gut axis hormones: glucagon-like peptide-1 (GLP-1), peptide YY (PYY), leptin, and ghrelin.

In the period from April 2013 to January 2014, 35 people meeting the inclusion criteria and who gave their informed consent for participation in the study were enrolled in the study, selected from 51 patients treated for super obesity at the $2^{\text {nd }}$ Chair of Surgery, JUMC. The patients were qualified for specific surgical procedures. Then, the group of volunteers were covered by a prospective analysis. Fourteen (40\%) men and 21 (60\%) women gave their consent to participate in the study. The mean age of patients enrolled in the study was 42.75 years (44.4 for women, 41.1 for men). In the studied group the mean body weight before the surgery was $146.2 \mathrm{~kg}$ and the mean BMI index was $50.44 \mathrm{~kg} / \mathrm{m}^{2}$. Before the surgery, 15 (42.8\%) patients were diagnosed with hypertension, and $6(17.2 \%)$ patients were diagnosed with type 2 diabetes. Laparoscopic sleeve gastrectomy was performed in 16 (45.8\%) patients, and laparoscopic gastric bypass was performed in the remaining 19 (54.2\%) patients. Of the studied patients, $7 \mathrm{did}$ not come to a follow-up visit 12 months after the surgical treatment (4 LSG and 3 Roux-en-Y gastric bypass (LRYGB) patients). The final analysed group consisted of 28 patients (Figure 1 ).

The selected parameters were reassessed during the follow-up visit. Also material for determination of selected biochemical parameters was collected. When the whole material was collected, it was delivered to the Diagnostic Laboratory at the Chair of Clinical Biochemistry, JUMC, where specific parameters were determined. Characteristics of the studied group are shown in Table I. 


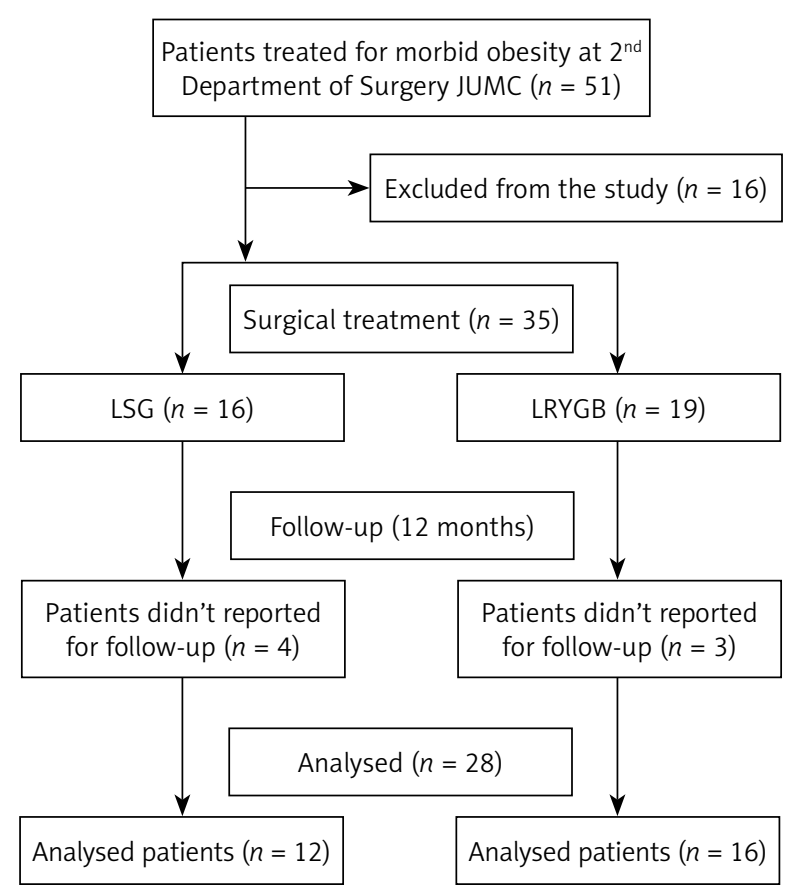

Figure 1. Study plan (flow chart)

\section{Description of laboratory procedures applied}

Leptin, ghrelin, GLP-1 and PYY levels were determined with commercially available reagent kits from Millipore, St. Charles, U.S. Leptin serum levels were determined with the immunoenzymatic technique ELISA using the Human Leptin ELISA Kit. The method was characterised by sensitivity of $0.78 \mathrm{ng} / \mathrm{ml}$, and inter- and intra-assay coefficients of variability below $4.6 \%$ and $6.2 \%$, respectively.

Ghrelin levels were determined with the immunoenzymatic technique using the Human Ghrelin (Total) ELISA Kit. The method determined two ghrelin forms: active and des-octanoyl. The method sensitivity limit corresponded to $0.50 \mathrm{pg} / \mathrm{ml}$, and inter- and intra-assay coefficients of variability were below $1.91 \%$ and $7.81 \%$, respectively. The serum levels of active GLP-1 were determined with the immunoenzymatic technique based on fluorescence detection, using fluorogenic substrate for alkaline phosphatase, 4-methylumbelliferyl phosphate. The Glucagon-Like Peptide-1 (Active) ELISA Kit used was characterised by sensitivity of 2 pM and inter- and intra-assay coefficients of variability below $9 \%$ and $13 \%$, respectively. Peptide $Y Y$ levels were determined with the immunoenzymatic technique using the $\mathrm{Hu}$ man PYY (Total) ELISA Kit. The analytical sensitivity of this method was $16.07 \mathrm{pg} / \mathrm{ml}$, while inter- and intra-assay coefficients of variability did not exceed $5.78 \%$ and $16.50 \%$, respectively.

\section{Statistical analysis}

The statistical analysis was conducted with the Statistica $10 \mathrm{PL}$ package. The two groups (according to the procedure type) were compared with the Student $t$-test and, when assumptions for standard distribution were not met, with the Mann-Whitney test. To compare changes in time, an analysis of variance

Table I. Detailed characteristics of the studied group

\begin{tabular}{|c|c|c|c|c|c|}
\hline Parameter & Gender & Sleeve gastrectomy (LSG) & Gastric bypass (LRYGB) & \multicolumn{2}{|c|}{ Total } \\
\hline \multirow[t]{2}{*}{ Gender } & Female & 8 & 9 & 17 & \multirow{2}{*}{28} \\
\hline & Male & 4 & 7 & 11 & \\
\hline \multirow[t]{2}{*}{ Age [years] } & Female & 43.22 & 45.87 & 44.4 & \multirow{2}{*}{42.75} \\
\hline & Male & 40.7 & 42.9 & 41.1 & \\
\hline \multirow[t]{2}{*}{ Weight [kg] } & Female & 134.8 & 141.1 & 137.95 & \multirow{2}{*}{146.2} \\
\hline & Male & 157.75 & 160.5 & 159.12 & \\
\hline \multirow[t]{2}{*}{$\mathrm{BMI}\left[\mathrm{kg} / \mathrm{m}^{2}\right]$} & Female & 50.66 & 51.5 & 51.03 & \multirow{2}{*}{50.44} \\
\hline & Male & 48.74 & 49.76 & 49.29 & \\
\hline \multirow[t]{2}{*}{ Hypertension } & Female & 2 & 7 & 9 & \multirow{2}{*}{15} \\
\hline & Male & 2 & 4 & 6 & \\
\hline \multirow[t]{2}{*}{ Type 2 diabetes } & Female & 0 & 1 & 1 & \multirow{2}{*}{6} \\
\hline & Male & 2 & 3 & 5 & \\
\hline
\end{tabular}


with repeated measurements was used, and when the criteria were not met, relevant nonparametric tests (Friedman test, Wilcoxon signed-rank test) were used. In all analyses, effects for which the probability value, $p$, was below the adapted level of significance $\alpha=0.05(p<0.05)$ were considered significant.

\section{Bioethics committee}

The study was approved by the Bioethics Committee, Jagiellonian University. After getting thoroughly acquainted with assumptions and objectives of the study, all patients enrolled in the study group gave their written informed consent to participate in the study.

\section{Results}

The mean body weight measured during the follow-up visit 12 months after the surgery was $97.1 \mathrm{~kg}$. The mean weight was $92.8 \mathrm{~kg}$ in the group of patients after LSG and $101.9 \mathrm{~kg}$ in the group after laparoscopic gastric bypass $(p=0.52)$. The mean BMI after the surgical procedure was $33.4 \mathrm{~kg} / \mathrm{m}^{2}$, and $32.5 \mathrm{~kg} / \mathrm{m}^{2}$ and $34.4 \mathrm{~kg} / \mathrm{m}^{2}$ in the group after LSG and laparoscopic gastric bypass, respectively $(p=0.32)$. The average percentage weight loss (\%EWL) was $58.8 \%$, and the average percentage excess BMI loss (\%EBMIL) was $68.85 \%$. One year after the surgical procedure no significant differences were noted between the procedures in terms of body mass reduction rate (Table II).

From 15 patients diagnosed with hypertension before the surgical procedure, in 11 (73\%) no clinical signs of hypertensions were observed during the follow-up visit 12 months after the procedure, and in the remaining patients the doses of hypotensive medicines were reduced. Significant improvement was also observed for type 2 diabetes, versus the period before the surgery. Only in 1 of 6 patients diagnosed with type 2 diabetes before the bariatric procedure were the symptoms of sugar management disorder maintained during the follow-up visit. This means that in 5 (83.3\%) of the patients diabetes resolved with applied treatment.

Table II. Body mass reduction rate depending on type of surgical procedure

\begin{tabular}{|lccc|}
\hline & LSG & LRYGB & Value of $p$ \\
\hline \%EWL & $61.2 \%$ & $56.19 \%$ & 0.1 \\
\hline \%EBMIL & $71.8 \%$ & $65.5 \%$ & 0.09 \\
\hline
\end{tabular}

When analysing results for activity levels of selected incretins and gastrointestinal tract hormones, statistically significant differences were observed for ghrelin, leptin and PYY levels. Observed changes in GLP-1 levels were not statistically significant (Figure 2).

In the whole studied group, the ghrelin level dropped from the mean level of $350.2 \mathrm{pg} / \mathrm{ml}$ to $100.34 \mathrm{pg} / \mathrm{ml}(p=0.003)$. The ghrelin level decreased from $384.6 \mathrm{pg} / \mathrm{ml}$ to $105.5 \mathrm{pg} / \mathrm{ml}(0.024)$ in patients treated with the laparoscopic sleeve gastrectomy, and from $337.3 \mathrm{pg} / \mathrm{ml}$ to $98.36 \mathrm{pg} / \mathrm{ml}$ (0.025) in patients treated with the laparoscopic gastric bypass. No statistically significant difference was observed between those two procedures (Figure 3).

A significant drop was also observed for leptin levels $(18.4 \mathrm{ng} / \mathrm{ml})$ vs. its levels before the surgery $(55.7 \mathrm{ng} / \mathrm{ml})$. The obtained result is statistically significant $(p=0.005)$. The results were similar for both types of procedure, and no differences were demonstrated between them. The leptin level decreased from $48.6 \mathrm{ng} / \mathrm{ml}$ to $9.78 \mathrm{ng} / \mathrm{ml}(p=0.023)$ for the laparoscopic sleeve gastrectomy, and from $58.4 \mathrm{ng} /$ $\mathrm{ml}$ to $21.6 \mathrm{ng} / \mathrm{ml}(p=0.035)$ in patients treated with the laparoscopic gastric bypass (Figure 4).

When analysing results concerning PYY levels, a statistically significant increase was observed in the whole studied group, from $116.6 \mathrm{pg} / \mathrm{ml}$ to $182.9 \mathrm{pg} / \mathrm{ml}(p=0.01)$. Also for PYY the changes observed after both surgical procedures were statistically significant. The PYY level increased from

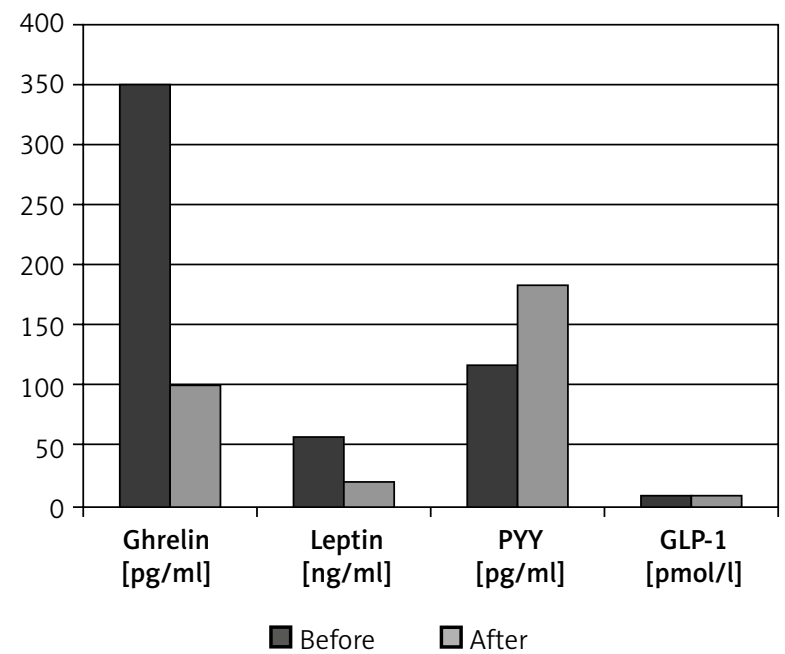

Figure 2. Changes in activity levels of selected incretins and gastrointestinal tract hormones following surgical treatment for super obesity 


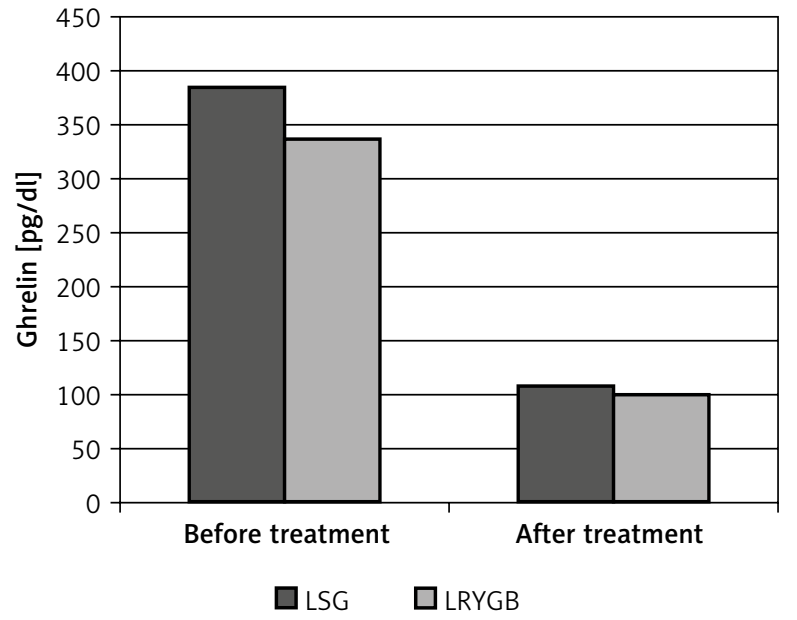

Figure 3. Change in ghrelin levels following a given type of surgical procedure

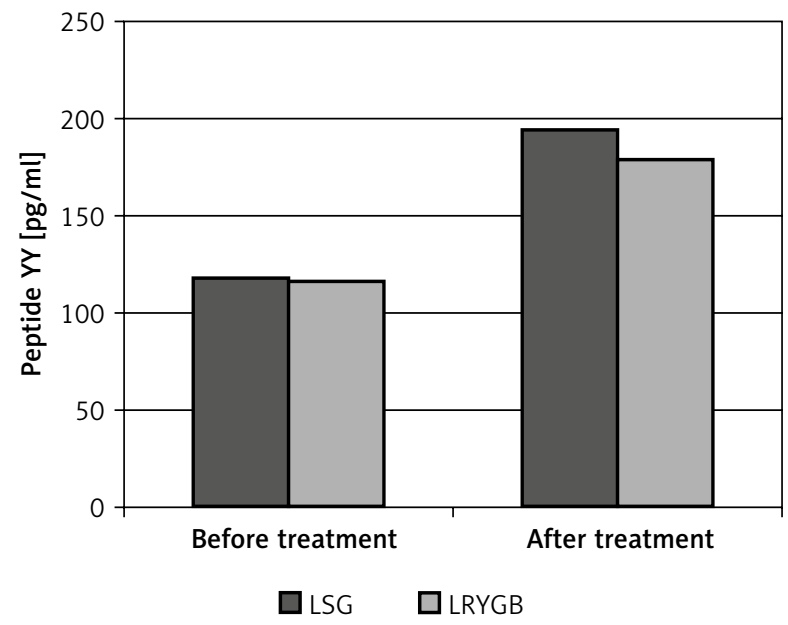

Figure 5. Change in PYY levels following a given type of surgical procedure

$117.6 \mathrm{pg} / \mathrm{ml}$ to $193.8 \mathrm{pg} / \mathrm{ml}(p=0.031)$ for the laparoscopic sleeve gastrectomy, and from $116.19 \mathrm{pg} /$ $\mathrm{ml}$ to $178.8 \mathrm{pg} / \mathrm{ml}(p=0.037)$ for the laparoscopic gastric bypass (Figure 5 ).

After the surgical treatment, the mean GLP-1 level increased from $5.6 \mathrm{pmol} / \mathrm{l}$ to $6.49 \mathrm{pmol} / \mathrm{l}$. However, the observed change was not statistically significant, both in the whole studied group $(p=0.42)$ and for individual types of surgical procedure (LSG $p=$ 0.4 and LRYGB $p=0.46$ ) (Figure 6). Detailed values of the studied parameters are provided in Table III.

\section{Discussion}

In medical circles, interest in surgical treatment of obesity is huge, and this is reflected in the num-

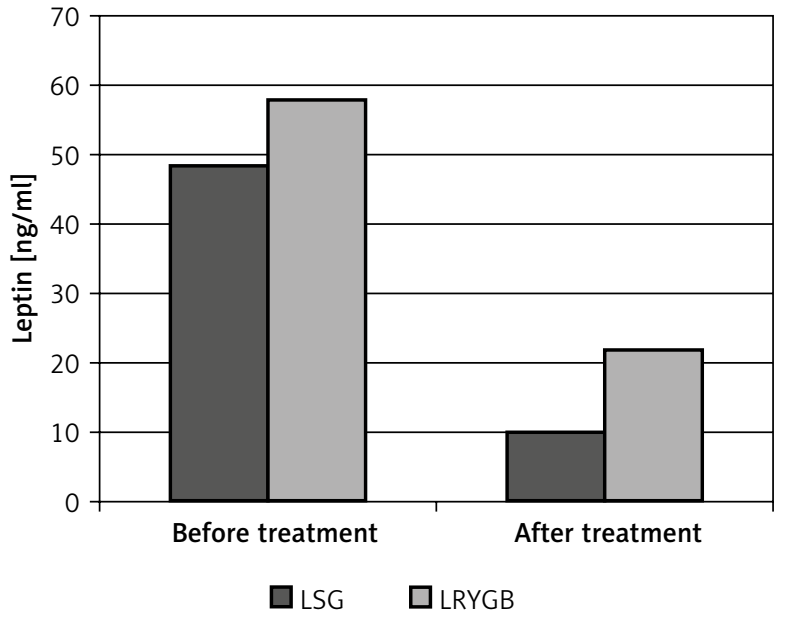

Figure 4. Change in leptin levels following a given type of surgical procedure

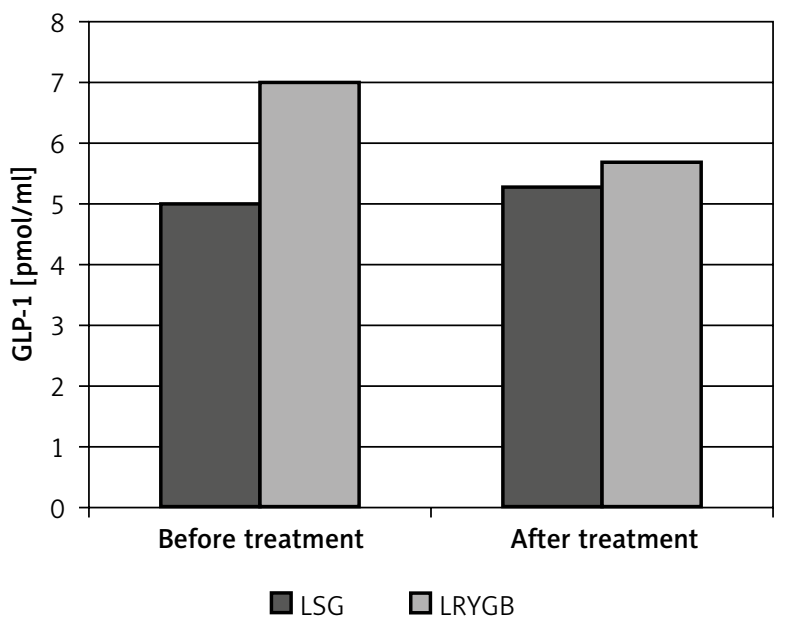

Figure 6. Change in GLP-1 levels following a given type of surgical procedure

ber of conducted studies and resultant publications. Bariatric surgery ceased to be just a method for treatment of obesity. Increasingly often it is emphasised that its main aim is treatment of comorbidities through its influence on patient's metabolism. Thus it was given another name "metabolic surgery".

Although metabolic effects of bariatric procedures seem to be relatively well known, still many aspects remain unclear or doubtful, or even opinions on them found in the literature are contradictory. One of the bariatric procedures with well-documented and explained effects is the gastric bypass, currently the most commonly performed bariatric procedure in the world. Its influence on metabolism of operated patients through hormonal changes oc- 
Table III. Detailed results of activity determinations for selected incretins and gastrointestinal tract hormones in patients treated for super obesity

\begin{tabular}{|lcccc|}
\hline Parameter & Procedure & Before the procedure & After the procedure & Value of $p$ \\
\hline Ghrelin $[\mathrm{pg} / \mathrm{ml}]$ & LSG & 384.6267 & 105.5967 & 0.024 \\
\cline { 2 - 4 } & LRYGB & 337.3025 & 98.36875 & 0.025 \\
\hline Leptin $[\mathrm{ng} / \mathrm{ml}]$ & LSG & 48.65833 & 9.781667 & 0.023 \\
\cline { 2 - 4 } & LRYGB & 58.43813 & 21.63375 & 0.035 \\
\hline PYY $[\mathrm{pg} / \mathrm{ml}]$ & LSG & 117.6967 & 193.815 & 0.031 \\
\cline { 2 - 4 } & LRYGB & 116.1913 & 178.8975 & 0.037 \\
\hline GLP-1 $[\mathrm{pmol} / \mathrm{ml}]$ & LSG & 5.057 & 5.325 & 0.4 \\
\cline { 2 - 4 } & LRYGB & 7.030 & 5.706 & 0.46 \\
\hline
\end{tabular}

curring directly after the surgery is a subject of numerous publications and reports.

However, current tendencies show a constant increase in interest in the LSG, and in the number of procedures conducted with this technique. It is know that sleeve gastrectomy is an effective and relatively safe procedure [7]. The achieved body mass reduction, previously considered to be solely the effect of the restrictive mechanism reducing possibilities for food supply, is currently questioned. The opinion that LSG also results in a metabolic response appears with increasing frequency. While the influence of this treatment method on postoperative reduction in the ghrelin level and a resultant decrease of appetite seems obvious, because the main source of ghrelin is cells in the fundus removed during the procedure, its influence on the other components controlling the brain-gut axis remains unclear [8].

The present study was designed to evaluate the metabolic effect of the two bariatric procedures currently most frequently performed in the world, that is, the LSG and the laparoscopic gastric bypass. The answer to this question may be a valuable guideline when qualifying patients with super obesity for specific surgical procedures.

The results of activity analyses of selected incretins and gastrointestinal tract hormones showed statistically significant differences for ghrelin, leptin and PYY levels. What is interesting, the observed changes in GLP-1 levels were not statistically significant.

The observed reduction in the ghrelin levels in patients after LSG is not surprising, and that effect was expected even before the study was conducted.
This effect results from the fact that the number of cells producing this hormone is reduced with removal of a fragment of the resected stomach including its fundus $[9,10]$. It was surprising, however, that in this study a significant reduction in the ghrelin level was also noted for the laparoscopic gastric bypass, comparable to the laparoscopic sleeve gastrectomy. In the gastric bypass procedure the main fraction of cells producing ghrelin is in the inactive, bypassed part of the stomach, and this possibly contributes to the reduction in its blood levels. An additional implied mechanism underlying a decrease in ghrelin secretion after gastric bypass surgery is a lack of mechanical stretching of the fundus by food, as it does not enter this part of the gastrointestinal tract after the surgery. Similar conclusions can be drawn when analysing results of Nanniperii's study, in which a significant reduction in ghrelin levels was also observed for both types of surgical procedure [11].

A reduction in body mass is accompanied by a reduction in fatty tissue in the body. Therefore, the number of adipocytes constituting a source of leptin decreases, and this leads to a reduction in blood levels of this peptide. In the conducted study, a significant reduction in the leptin level was observed for both surgical techniques. However, in the authors' opinion this effect is not directly related to changes in the hormonal axes, but rather results directly from the weight loss. In this study both types of surgery led to a comparable reduction in body weight. In both cases similar changes were also observed for the leptin levels. As expected, no significant differences were noted between the surgery procedures. The reduction in the leptin levels after 
bariatric treatment was also observed by Buchwald [12]. Similarly, Terra, in her work on delayed effects of the LSG and the laparoscopic gastric bypass, observed a significant drop in the leptin levels for both procedures [13].

When analysing literature data at the stage of planning of this study, an increase in PYY and GLP-1 levels was expected following the LRYGB procedure. The authors were surprised to see the increase in levels of incretins secreted by $L$ cells in jejunum after both surgical procedures. For PYY, the increase in its levels appeared to be significant $(p=0.01)$ one year after bariatric treatment. Therefore, both the LSG and the laparoscopic gastric bypass resulted in a similar increase in peptide YY levels. For LSG this effect is possibly related to the post-treatment change in the gastric emptying mechanism, so gastric content is moved to the duodenum much faster, thus stimulating the L cells. Although the mean GLP-1 level after both surgical procedures changed slightly in both groups, no statistical significance was noted for observed changes. In her study Rosa Morinigo documented a change in the GLP-1 activity level both 6 weeks after the gastric bypass surgery and during a follow-up visit 12 months after discharge from the hospital [14]. Therefore, there are reports available in the literature confirming the increase in the GLP-1 level after gastric bypass surgery. Its increase and influence on the increased post-meal insulin secretion from pancreatic beta cells is the main suggested mechanism underlying resolution of type 2 diabetes after this surgical procedure. Some studies also aimed at comparing individual types of surgical procedures in terms of their possible effect on levels of selected gastrointestinal hormones, including PYY and GLP-1. For example, in his paper Rainehr described a significant increase in PYY and GLP-1 when comparing the effects of the gastric bypass and gastric banding surgery. The observed change was significantly higher after the gastric bypass [15]. However, studies comparing the gastric bypass with the LSG remain scarce. One such study is the paper of Yousseif, who demonstrated a significant increase in PYY and GLP-1 levels after both of these surgical procedures. However, in patients after the LSG the levels of studied proteins were slightly lower during a follow-up [16]. It seems that the lack of statistically significant increase in GLP-1 levels after surgical treatment observed in this study may result from a low number of patients enrolled in the study. No significant results were observed in both groups of patients, operated with the gastric bypass technique and with the LSG technique. Therefore, it seems impossible to draw conclusions on possible differences between the procedures.

\section{Conclusions}

The present study and data available in the literature unambiguously indicate that surgical treatment for super obesity, either with the laparoscopic gastric bypass or with the LSG, leads to effective body mass reduction. After those two procedures an improvement in diseases caused by obesity (hypertension and type 2 diabetes) is observed. Both treatment methods result in modification of secretion patterns for selected gastrointestinal hormones, and this was considered to be a beneficial effect of bariatric treatment. The laparoscopic sleeve gastrectomy, being a procedure resulting in a metabolic response, seems to be an equally effective method for treatment of super obesity and comorbidities as the laparoscopic gastric bypass.

\section{Acknowledgments}

The publication of this paper was supported by the Faculty of Medicine, Jagiellonian University Medical College, Leading National Research Centre (KNOW) 2012-2017.

\section{Conflict of interest}

The authors declare no conflict of interest.

\section{References}

1. Maggard MA, Shugarman LR, Suttorp M, et al. Meta-analysis: surgical treatment of obesity. Ann Intern Med 2005; 142: 547-59.

2. Buchwald H, Estok R, Fahrbach K, et al. Trends in mortality in bariatric surgery: a systematic review and meta-analysis. Surgery 2007; 142: 621-32; discussion 632-5.

3. Hameed S, Dhillo WS, Bloom SR. Gut hormones and appetite control. Oral Dis 2009; 15: 18-26.

4. Buchwald H, Avidor Y, Braunwald E, et al. Bariatric surgery: a systematic review and meta-analysis. JAMA 2004; 292: 1724-37.

5. Pournaras DJ, Le Roux CW. The effect of bariatric surgery on gut hormones that alter appetite. Diabetes Metab 2009; 35: 508-12.

6. Pournaras DJ, le Roux CW. Obesity, gut hormones, and bariatric surgery. World J Surg 2009; 33: 1983-8.

7. Szewczyk T, Janczak P, Janiak A, et al. Laparoscopic sleeve gastrectomy -7 years of own experience. Videosurgery Miniinv 2014; 9: 427-35. 
8. Lehmann A, Bobowicz M, Lech P, et al. Comparison of percentage excess weight loss after laparoscopic sleeve gastrectomy and laparoscopic adjustable gastric banding. Videosurgery Miniinv 2014; 9: 351-6.

9. Buzga M, Zavadilová V, Holéczy P, et al. Dietary intake and ghrelin and leptin changes after sleeve gastrectomy. Videosurgery Miniinv 2014; 9: 554-61.

10. Kasalicky M, Dolezel R, Vernerova E, Haluzik M. Laparoscopic sleeve gastrectomy without over-sewing of the staple line is effective and safe. Videosurgery Miniinv 2014; 9: 46-52.

11. Nannipieri M, Baldi S, Mari A, et al. Roux-en-Y gastric bypass and sleeve gastrectomy: mechanisms of diabetes remission and role of gut hormones. J Clin Endocrinol Metab 2013; 98: 4391-9.

12. Buchwald H, Dorman RB, Rasmus NF, et al. Effects on GLP-1, PYY, and leptin by direct stimulation of terminal ileum and cecum in humans: implications for ileal transposition. Surg Obes Relat Dis 2014; 10: 780-6.

13. Terra X, Auguet T, Guiu-Jurado E, et al. Long-term changes in leptin, chemerin and ghrelin levels following different bariatric surgery procedures: Roux-en-Y gastric bypass and sleeve gastrectomy. Obes Surg 2013; 23: 1790-8.

14. Morinigo R, Lacy AM, Casamitjana R, et al. GLP-1 and changes in glucose tolerance following gastric bypass surgery in morbidly obese subjects. Obes Surg 2006; 16: 1594-601.

15. Reinehr T, Roth CL, Schernthaner GH, et al. Peptide YY and glucagon-like peptide-1 in morbidly obese patients before and after surgically induced weight loss. Obes Surg 2007; 17: 1571-7.

16. Yousseif A, Emmanuel J, Karra E, et al. Differential effects of laparoscopic sleeve gastrectomy and laparoscopic gastric bypass on appetite, circulating acyl-ghrelin, peptide YY3-36 and active GLP-1 levels in non-diabetic humans. Obes Surg 2014; 24: $241-52$

Received: 23.04.2015, accepted: 8.06.2015. 